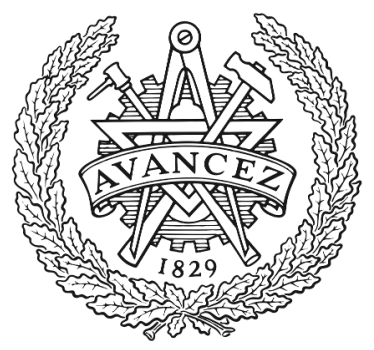

CHALMERS

UNIVERSITY OF TECHNOLOGY

\title{
Distributed Two-Way Localization Bounds for 5G mmWave Systems
}

Downloaded from: https://research.chalmers.se, 2023-04-26 09:30 UTC

Citation for the original published paper (version of record):

Abu-Shaban, Z., Wymeersch, H., Abhayapala, T. et al (2019). Distributed Two-Way Localization Bounds for 5G mmWave Systems. GLOBECOM - IEEE Global Telecommunications Conference. http://dx.doi.org/10.1109/GLOCOMW.2018.8644178

N.B. When citing this work, cite the original published paper. 


\title{
Distributed Two-Way Localization Bounds for 5G mmWave Systems
}

\author{
Zohair Abu-Shaban*, Henk Wymeersch ${ }^{\dagger}$, Thushara Abhayapala ${ }^{\ddagger}$, Gonzalo Seco-Granados ${ }^{\S}$ \\ *University of New South Wales Canberra, Australia. Email: zohair.abushaban@ieee.org \\ †Chalmers University of Technology, Sweden. Email: henkw@chalmers.se \\ $\ddagger$ The Australian National University, Australia. Email: thushara.abhayapala@anu.edu.au \\ §Universitat Autònoma de Barcelona, Spain.Email: gonzalo.seco@uab.es
}

\begin{abstract}
Recently, localization for 5G millimeter-wave communication systems has been shown to provide highaccuracy performance, with error being in the order of tens of centimeters. However, most of the literature assumes a high level of synchronization, which is not always the case practically. To address this matter, we investigate a distributed two-way localization protocol (DLP) that relieves the need for tight timing synchronization. We derive the position and orientation bounds, when localization is initiated and carried out by the base station. Our simulation results show that the performance of DLP is identical to that of the synchronized one-way localization. We thus conclude that the considered 5G localization is limited by the estimation of the angles rather than the delay. The results also imply that orientation estimation is more challenging than position estimation.
\end{abstract}

\section{INTRODUCTION}

With the high localization accuracy of $5 \mathrm{G}$ millimeterwave (mmWave) communication systems, location-aided communication is becoming more attractive. The location information creates two kinds of opportunities unique to $5 \mathrm{G}$ mmWave, since it enables location-aware applications [1], [2], e.g., vehicular communication, and assisted living systems, while also supporting communication systems design and optimization [3]-[5] such as beamforming, pilot assignment, and resources allocation.

Due to the utilization of arrays with high number of antennas at the transmitter and the receiver, and the allocation of massive bandwidths, spatiotemporal localization with single base station (BS) can be seen as the ultimate localization strategy for $5 \mathrm{G}$. With the high number of antennas, the directions of arrival (DOA) and departure (DOD) can be estimated with very low error [6], while the large bandwidth enables highly accurate estimation of the time of arrival (TOA) [7]. Subsequently, combining the spatial and temporal estimates, the user equipment (UE) location can be determined.

The accuracy of single-anchor localization for $5 \mathrm{G}$ mmWave systems has been studied recently by several papers in terms of position (PEB) and orientation error bounds (OEB). The UE PEB and OEB of 2D localization in $5 \mathrm{G}$ mmWave systems were investigated in [8] using uniform linear arrays. On the other hand, the PEB and OEB for mmWave 3D localization were derived with different approaches in [9] and [10] using arrays with arbitrary geometries. The results in [8]-[10] showed that the error performance of $5 \mathrm{G} \mathrm{mmWave} \mathrm{localization} \mathrm{is} \mathrm{in}$ the order of centimeters. However, one important, yet usually overlooked, requirement for localization is the synchronization of BS and UE. For example, [8] and [10] assume that the BS and UE are perfectly synchronized, while [9] assumes coarse synchronization, and includes a residual synchronization error in the model.

Motivated by two-way ranging that use the time-offlight [11], in this paper, we focus on two-way localization (TWL). We study the PEB and OEB under lineof-sight (LOS) communication with a distributed TWL protocol (DLP) that accounts for timing bias between the clocks of the BS and UE. Higher order artifacts such as clock drift and skew are not addressed herein, but can be estimated using three- or multi-way ranging [11], [12]. Under DLP, the BS initiates the localization process by transmitting a known signal to a UE. Then, the UE estimates the TOA with reference to its local clock and transmits back another known signal, after a preagreed waiting interval. Subsequently, upon receiving the UE signal, the BS estimates the range between the two devices with reference to its local clock. Since this clock was initially used to transmit the first signal, full time synchronization between UE and BS is not needed. Eventually, localization is achieved at the BS, using the signal received back after the second transmission round.

Considering localization at BS only, we investigate the DLP for LOS 5G mmWave signals, as a means of alleviating the tight synchronization requirement of localization. Towards that, we consider the timing bias between the BS and UE as a nuisance parameter and derive the Fisher information matrices (FIM) of the position and orientation. Based on these FIMs, we evaluate the PEB and OEB numerically, and compare them to those of the traditional one-way localization (OWL) [10]. The results provided herein are part of an extensive study presented in [13], where both network-side and device-side localization are investigated for distributed and centralized TWL protocols.

\section{Channel Model And Beamforming}

Consider uplink localization, whereby a BS, located at the origin of the $3 \mathrm{D}$ space with zero orientation angles, attempts to estimate the UE position, $\mathbf{p} \triangleq\left[p_{x}, p_{y}, p_{z}\right]^{\mathrm{T}}$, and orientation, $\mathbf{o} \triangleq\left[\zeta_{0}, \chi_{0}\right]^{\mathrm{T}}$. Similar to [10], we define $\zeta_{0}$ as the rotation angle around the $z$-axis, which yields new coordinate axes $x^{\prime}, y^{\prime}$ and $z$, while $\chi_{0}$ is defined 

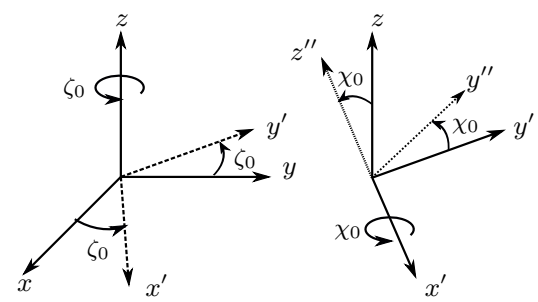

Fig. 1. Two-step rotation: First around $z$-axis, then around $x^{\prime}$-axis. as the rotation angle around the $x^{\prime}$-axis. See Fig. 1. This is representative of practical applications characterized by two rotation angles, such as vehicular and robotic applications ${ }^{1}$. Both BS and UE are equipped with antenna arrays of arbitrary but known geometries and communicate through a LOS. In this context, we assume that the NLOS parameters, if any, can be estimated as in [10]. However, unlike [10], we use only the DOA, DOD and TOA of the LOS path to compute the PEB and OEB. Consequently, for clarity, we can write the signal model explicitly by including only the $\operatorname{LOS}^{2}$.

In the following, the parameters related to BS and UE are denoted by the subscripts " $B$ " and " $U$ ", respectively, while the superscripts "f" and "b" denote the parameters to the forward and backward transmissions, respectively.

1) Forward Channel: The forward signal, received at UE at time $t=\tau^{\mathrm{f}}$, undergoes a channel given by

$$
\mathbf{H}^{\mathrm{f}}(\beta, \boldsymbol{\vartheta}) \triangleq \mathbf{H}_{\mathrm{s}}^{\mathrm{f}}(\beta, \boldsymbol{\vartheta}) \delta\left(t-\tau^{\mathrm{f}}\right), \in \mathbb{C}^{N_{\mathrm{U}} \times N_{\mathrm{B}}}
$$

where $\mathbf{H}_{\mathrm{S}}^{\mathrm{f}}(\beta, \boldsymbol{\vartheta}) \triangleq \sqrt{N_{\mathrm{B}} N_{\mathrm{U}}} \beta \mathbf{a}_{\mathrm{U}}\left(\theta_{\mathrm{U}}, \phi_{\mathrm{U}}\right) \mathbf{a}_{\mathrm{B}}^{\mathrm{H}}\left(\theta_{\mathrm{B}}, \phi_{\mathrm{B}}\right)$ is the channel part corresponding to the spatial channel parameters, such that $\beta$ is the complex path gain, $N_{\mathrm{B}}$ and $N_{\mathrm{U}}$ are the number of antennas at $\mathrm{BS}$ and $\mathrm{UE}$, respectively, and

$$
\vartheta \triangleq\left[\theta_{\mathrm{B}}, \phi_{\mathrm{B}}, \theta_{\mathrm{U}}, \phi_{\mathrm{U}}\right]^{\mathrm{T}},
$$

and $\left(\theta_{\mathrm{U}}, \phi_{\mathrm{U}}\right)$ and $\left(\theta_{\mathrm{B}}, \phi_{\mathrm{B}}\right)$ are the forward DOAs and DODs. Finally, $\mathbf{a}_{\mathrm{U}}$ and $\mathbf{a}_{\mathrm{B}}$ are the array response vectors

$$
\begin{array}{ll}
\mathbf{a}_{\mathrm{U}}\left(\theta_{\mathrm{U}}, \phi_{\mathrm{U}}\right) \triangleq \frac{1}{\sqrt{N_{\mathrm{U}}}} e^{-j \boldsymbol{\Delta}_{\mathrm{U}}^{\mathrm{T}} \mathbf{k}\left(\theta_{\mathrm{U}}, \phi_{\mathrm{U}}\right)}, & \in \mathbb{C}^{N_{\mathrm{U}}} \\
\mathbf{a}_{\mathrm{B}}\left(\theta_{\mathrm{B}}, \phi_{\mathrm{B}}\right) \triangleq \frac{1}{\sqrt{N_{\mathrm{B}}}} e^{-j \boldsymbol{\Delta}_{\mathrm{B}}^{\mathrm{T}} \mathbf{k}\left(\theta_{\mathrm{B}}, \phi_{\mathrm{B}}\right)}, & \in \mathbb{C}^{N_{\mathrm{B}}}
\end{array}
$$

where $\mathbf{k}(\theta, \phi)=\frac{2 \pi}{\lambda}[\cos \phi \sin \theta, \sin \phi \sin \theta, \cos \theta]^{\mathrm{T}}$ is the wavenumber vector, $\lambda$ is the wavelength, $\boldsymbol{\Delta}_{\mathrm{B}} \in \mathbb{C}^{3 \times N_{\mathrm{B}}}$ is a matrix whose columns contain the $3 \mathrm{D}$ Cartesian coordinates of the array elements of BS in meters, and $\boldsymbol{\Delta}_{\mathrm{U}} \in \mathbb{C}^{3 \times N_{\mathrm{U}}}$ is defined similarly for UE. For presentation purposes, we drop the angle parameters from the notation of $\mathbf{a}_{\mathrm{B}}$ and $\mathbf{a}_{\mathrm{U}}$.

The signal transmitted from $\mathrm{BS}$ is modeled by $\sqrt{E_{\mathrm{t}}} \mathbf{F}_{\mathrm{B}} \mathbf{S}_{\mathrm{B}}(t)$, where $E_{\mathrm{t}}$ is the transmitted energy per symbol, and $\mathbf{F}_{\mathrm{B}} \triangleq\left[\mathbf{f}_{\mathrm{B}, 1}, \mathbf{f}_{\mathrm{B}, 2}, \ldots \mathbf{f}_{\mathrm{B}, N_{\mathrm{b}_{\mathrm{B}}}}\right]$ is the BS transmit beamforming matrix, $\mathbf{f}_{\mathrm{B}, b}, 1 \leq b \leq N_{\mathrm{b}_{\mathrm{B}}}$ is the $b^{\text {th }}$

\footnotetext{
${ }^{1}$ This corresponds for instance to a vehicle that can turn left and right $\left(\zeta_{0}\right)$ or ascend and descend $\left(\chi_{0}\right)$, but does not slip or flip.

${ }^{2}$ The protocols presented in this paper could also exploit the reflected components for positioning. Conceptually, this can be done by applying the path orthogonality arguments presented in [10].
}

BS beam, and $N_{\mathrm{b}_{\mathrm{B}}}$ is the number of transmit beams. The pilot signal $\mathbf{s}_{\mathrm{B}}(t) \triangleq\left[s_{\mathrm{B}, 1}(t), s_{\mathrm{B}, 2}(t), \ldots, s_{\mathrm{B}, N_{\mathrm{b}_{\mathrm{B}}}}(t)\right]^{\mathrm{T}}$ has

$$
s_{\mathrm{B}, b}(t)=\sum_{\ell=0}^{N_{\mathrm{s}}-1} a_{\mathrm{B}, \ell}^{(b)} p\left(t-\ell T_{\mathrm{s}}\right), \quad 1 \leq b \leq N_{\mathrm{B}_{\mathrm{B}}},
$$

where $a_{\mathrm{B}, \ell}^{(b)}$ are known unit-energy pilot symbols transmitted over the $b^{\text {th }}$ beam from $\mathrm{BS}$, and $p(t)$ is a unit-energy pulse with a power spectral density (PSD), denoted by $|P(f)|^{2}$. In (5), $N_{\mathrm{s}}$ is the number of pilot symbols and $T_{\mathrm{s}}$ is the symbol duration, leading to a total observation time of $T_{\mathrm{o}} \approx N_{\mathrm{s}} T_{\mathrm{s}}$. Note that we keep the transmitted power fixed with $N_{\mathrm{b}_{\mathrm{B}}}$ by setting $\operatorname{Tr}\left(\mathbf{F}_{\mathrm{B}}^{\mathrm{H}} \mathbf{F}_{\mathrm{B}}\right)=1$, and $\mathbf{s}_{\mathrm{B}}(t) \mathbf{s}_{\mathrm{B}}^{\mathrm{H}}(t)=\mathbf{I}_{N_{\mathrm{b}_{\mathrm{B}}}}$, where $\operatorname{Tr}(\cdot)$ denotes the matrix trace, and $\mathbf{I}_{N_{\mathrm{b}_{\mathrm{B}}}}$ is the identity matrix.

Similarly, define the receive beamforming matrix at $\mathrm{UE}$ as $\mathbf{W}_{\mathrm{U}} \triangleq\left[\mathbf{w}_{\mathrm{U}, 1}, \mathbf{w}_{\mathrm{U}, 2}, \ldots \mathbf{w}_{\mathrm{U}, N_{\mathrm{b}_{\mathrm{U}}}}\right]$, where $\mathbf{w}_{\mathrm{U}, k}, 1 \leq k \leq N_{\mathrm{b}_{\mathrm{U}}}$ is a UE receive beam, and $N_{\mathrm{b}_{\mathrm{U}}}$ is the number of receive beams.

2) Backward Channel: Channel from UE to BS is

$$
\mathbf{H}^{\mathrm{b}}(\beta, \boldsymbol{\vartheta}) \triangleq \mathbf{H}_{\mathrm{s}}^{\mathrm{b}}(\beta, \boldsymbol{\vartheta}) \delta\left(t-\tau^{\mathrm{b}}\right) \in \mathbb{C}^{N_{\mathrm{B}} \times N_{\mathrm{U}}},
$$

where $\mathbf{H}_{\mathrm{s}}^{\mathrm{b}}(\beta, \boldsymbol{\vartheta}) \triangleq \sqrt{N_{\mathrm{B}} N_{\mathrm{U}}} \beta \mathbf{a}_{\mathrm{B}}\left(\theta_{\mathrm{B}}, \phi_{\mathrm{B}}\right) \mathbf{a}_{\mathrm{U}}^{\mathrm{H}}\left(\theta_{\mathrm{U}}, \phi_{\mathrm{U}}\right)$ such that $\tau^{\mathrm{b}}$ denotes the local TOA at BS, $\left(\theta_{\mathrm{U}}, \phi_{\mathrm{U}}\right)$ and $\left(\theta_{\mathrm{B}}, \phi_{\mathrm{B}}\right)$ are the backward DODs and DOAs at UE and $\mathrm{BS}$, respectively. We assume that both transmissions occur within the coherence time so that the channel gain remains unchanged. In the backward transmission, UE transmits with a beamforming matrix, $\mathbf{F}_{U}$ containing $N_{\mathrm{b}_{\mathrm{U}}}$ beams, while BS receives via a beamforming matrix, $\mathbf{W}_{\mathrm{B}}$ containing $N_{\mathrm{b}_{\mathrm{B}}}$ beams. Both $\mathbf{F}_{\mathrm{U}}$ and $\mathbf{W}_{\mathrm{B}}$ are defined similar to $\mathbf{W}_{\mathrm{U}}$ and $\mathbf{F}_{\mathrm{B}}$, respectively, but with possibly different beam directions.

Our objective is to derive the error bounds of estimating $\mathbf{p}$ and $\mathbf{o}$, from TOA, DOA, and DOD, obtained through DLP, in the presence of timing offset, $B$, and path gain, $\beta$, as nuisance parameters.

\section{Distributed Two-WAy Localization PROTOCOL}

In our formulation, we assume that the UE has a clock bias with respect to the BS clock, denoted by $B$. We denote the propagation delay (nominal TOA) by $\tau=$ $\|\mathbf{p}\| / c$, where $c$ is the speed of light. See Fig. 2 .

The forward transmission is initiated by the BS at time $t=0$, and received at $\mathrm{UE}$ at local time

$$
t=\tau^{\mathrm{f}}=B+\tau \text {. }
$$

Consequently, the observed signal after beamforming at the $\mathrm{UE}$ is given by

$$
\mathbf{y}_{\mathrm{U}}(t)=\sqrt{E_{\mathrm{t}}} \mathbf{W}_{\mathrm{U}}^{\mathrm{H}} \mathbf{H}_{\mathrm{s}}^{\mathrm{f}}(\beta, \boldsymbol{\vartheta}) \mathbf{F}_{\mathrm{B}} \mathbf{s}_{\mathrm{B}}\left(t-\tau^{\mathrm{f}}\right)+\mathbf{n}_{\mathrm{U}}(t),
$$

Denoting the real and imaginary parts of $\beta$ by $\beta_{\mathrm{R}}$ and $\beta_{\mathrm{I}}$, we find the FIM of $\left[\boldsymbol{\vartheta}^{\mathrm{T}}, \beta_{\mathrm{R}}, \beta_{\mathrm{I}}, \tau^{\mathrm{f}}\right]^{\mathrm{T}}$ based on $\mathbf{y}_{\mathrm{U}}(t)$.

On the other hand, the backward transmission is initiated by the UE at time $t=t^{\mathrm{b}}$, and received by $\mathrm{BS}$ at a local time

$$
t=\tau^{\mathrm{b}}=t^{\mathrm{b}}+\tau-B .
$$


After a pre-agreed delay $\tau^{\mathrm{D}}$, measured from the time $\mathbf{y}_{\mathbf{U}}(t)$ is received, UE sends back a signal $\mathbf{s}_{\mathbf{U}}(t)$ at

$$
t^{\mathrm{b}}=\hat{\tau}^{\mathrm{f}}+\tau^{\mathrm{D}}
$$

Subsequently, BS receives the signal $\mathbf{y}_{\mathrm{B}}(t)$ at

$$
\tau^{\mathrm{b}}=\hat{\tau}^{\mathrm{f}}+\tau^{\mathrm{D}}+\tau-B=2 \tau+e^{\mathrm{f}}+\tau^{\mathrm{D}} .
$$

Thus, the observed signal after beamforming at BS is

$$
\mathbf{y}_{\mathrm{B}}(t)=\sqrt{E_{\mathrm{t}}} \mathbf{W}_{\mathrm{B}}^{\mathrm{H}} \mathbf{H}_{\mathrm{s}}^{\mathrm{b}}(\beta, \boldsymbol{\vartheta}) \mathbf{F}_{\mathrm{U}} \mathbf{s}_{\mathrm{U}}\left(t-\tau^{\mathrm{b}}\right)+\mathbf{n}_{\mathrm{B}}(t)
$$

Based on $\mathbf{y}_{\mathrm{B}}(t)$, BS estimates $\hat{\tau}^{\mathrm{b}}$ and eventually determines $\mathbf{p}$, and $\mathbf{o}$. Note that $B$ in the forward and backward transmissions cancel out, and need not be estimated at UE. Thus, based on $\mathbf{y}_{\mathrm{B}}(t)$, the FIM of $\left[\boldsymbol{\vartheta}^{\mathrm{T}}, \beta_{\mathrm{R}}, \beta_{\mathrm{I}}, \tau^{\mathrm{b}}\right]^{\mathrm{T}}$ is determined.

Denoting the equivalent FIMs (EFIMs) of $\tau^{\mathrm{f}}$ and $\tau^{\mathrm{b}}$ by $J_{\tau^{\mathrm{f}}}$ and $J_{\tau^{\mathrm{b}}}$, respectively, we introduce the following estimation error notation

$$
e^{\mathrm{f}} \triangleq \hat{\tau}^{\mathrm{f}}-\tau^{\mathrm{f}}, \quad \text { and } \quad e^{\mathrm{b}} \triangleq \hat{\tau}^{\mathrm{b}}-\tau^{\mathrm{b}},
$$

such that

$$
\mathbb{E}\left\{\left(e^{\mathrm{f}}\right)^{2}\right\} \geq J_{\tau^{\mathrm{f}}}^{-1}, \quad \mathbb{E}\left\{\left(e^{\mathrm{b}}\right)^{2}\right\} \geq J_{\tau^{\mathrm{b}}}^{-1} .
$$

Finally, since the received signals are observed at the beamformer output, the noise terms in (8) and (11) are zero-mean additive spatially-correlated Gaussian noise. Therefore, the corresponding noise auto-covariance matrices are $\mathbf{R}_{\mathrm{n}_{\mathrm{U}}}=N_{0} \mathbf{W}_{\mathrm{U}}^{\mathrm{H}} \mathbf{W}_{\mathrm{U}}$, and $\mathbf{R}_{\mathrm{n}_{\mathrm{B}}}=N_{0} \mathbf{W}_{\mathrm{B}}^{\mathrm{H}} \mathbf{W}_{\mathrm{B}}$ where $N_{0}$ is the noise PSD, which is assumed to be identical at BS and UE.

\section{DERIVATION OF PEB AND OEB FOR DLP}

The PEB and OEB can be computed from the EFIM of position and orientation, $\mathbf{J}_{\mathbf{o}, \mathbf{p}}^{\mathrm{e}}$,

$$
\begin{aligned}
\mathrm{OEB} & \triangleq \sqrt{\left[\mathbf{J}_{\mathbf{o}, \mathbf{p}}^{\mathrm{e}}\right]_{1,1}+\left[\mathbf{J}_{\mathbf{o}, \mathbf{p}}^{\mathrm{e}}\right]_{2,2}}, \\
\mathrm{PEB} & \triangleq \sqrt{\left[\mathbf{J}_{\mathbf{o}, \mathbf{p}}^{\mathrm{e}}\right]_{3,3}+\left[\mathbf{J}_{\mathbf{o}, \mathbf{p}}^{\mathrm{e}}\right]_{4,4}+\left[\mathbf{J}_{\mathbf{o}, \mathbf{p}}^{\mathrm{e}}\right]_{5,5}} .
\end{aligned}
$$

Since $\mathbf{J}_{\mathbf{o}, \mathbf{p}}^{\mathrm{e}}$ is obtained by transforming the FIM of channel parameters, we start by computing this FIM. Then, we derive PEB and OEB using a parameter transformation procedure similar to [10].

1) FIM of Channel Parameters: In light of (6), (10) and (11), the vector of the unknowns under DLP is defined by

$$
\boldsymbol{\varphi}_{\mathrm{D}} \triangleq\left[\boldsymbol{\vartheta}^{\mathrm{T}}, \beta_{\mathrm{R}}, \beta_{\mathrm{I}}, \tau\right]^{\mathrm{T}} .
$$

Consequently, the FIM of $\varphi_{\mathrm{D}}$ is defined as

$$
\mathbf{J}_{\varphi_{\mathrm{D}}} \triangleq\left[\begin{array}{cc}
\mathbf{J}_{\mathrm{SS}}^{\mathrm{b}} & \mathbf{0}_{6} \\
\mathbf{0}_{6}^{\mathrm{T}} & J_{\tau \tau}
\end{array}\right] \text {, }
$$

where,

$$
\mathbf{J}_{\mathrm{SS}}^{\mathrm{b}}=\left[\begin{array}{cc}
\mathbf{J}_{\boldsymbol{\vartheta} \vartheta}^{\mathrm{b}} & \mathbf{J}_{\boldsymbol{\vartheta} \beta}^{\mathrm{b}} \\
\left(\mathbf{J}_{\boldsymbol{\vartheta} \beta}^{\mathrm{b}}\right)^{\mathrm{T}} & J_{\beta_{\mathrm{R}} \beta_{\mathrm{R}}}^{\mathrm{b}} \mathbf{I}_{2}
\end{array}\right],
$$

is the FIM corresponding to the spatial part of $\mathbf{J}_{\varphi_{\mathrm{D}}}$. The value of $J_{\tau \tau}$ as well as the entries of (18) are derived in the Appendix. Note that, as discussed in [10],

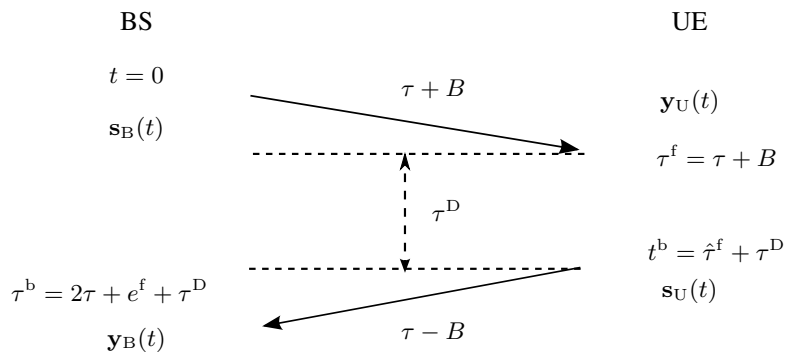

Fig. 2. The timeline of the distributed TWL protocol. the temporal and spatial parts in (17) are independent based on realistic mmWave assumptions: large number of transmit and receive antennas, large bandwidth and spatially sparse channel. Moreover, note that in (18), we used the fact that $J_{\beta_{\mathrm{R}} \beta_{\mathrm{R}}}^{\mathrm{b}}=J_{\beta_{\mathrm{I}} \beta_{\mathrm{I}}}^{\mathrm{b}}$.

While we can determine $J_{\tau^{\mathrm{f}}}$ based on $\mathbf{y}_{\mathrm{U}}(t)$, the FIM of $\left[\boldsymbol{\vartheta}^{\mathrm{T}}, \beta_{\mathrm{R}}, \beta_{\mathrm{I}}, \tau^{\mathrm{b}}\right]^{\mathrm{T}}$ is based on $\mathbf{y}_{\mathrm{B}}(t)$. Hence, to obtain the FIM of $\varphi_{\mathrm{D}}$ that includes $\tau$ rather than $\tau^{\mathrm{b}}$, we apply the fact that the delays are independent of any other parameter [10]. Towards that, recall that $\hat{\tau}^{\mathrm{b}}=2 \tau+e^{\mathrm{f}}+$ $e^{\mathrm{b}}+\tau^{\mathrm{D}}$, and define

$$
\tau^{\prime} \triangleq \frac{\hat{\tau}^{\mathrm{b}}-\tau^{\mathrm{D}}}{2}=\tau+\frac{e^{\mathrm{f}}+e^{\mathrm{b}}}{2} .
$$

Consequently, using (13) yields

$$
\mathbb{E}\left\{\left(\tau^{\prime}-\tau\right)^{2}\right\} \geq \frac{1}{4}\left(J_{\tau^{\mathrm{f}}}^{-1}+J_{\tau^{\mathrm{b}}}^{-1}\right)
$$

that is,

$$
J_{\tau \tau}=4\left(J_{\tau^{\mathrm{f}}}^{-1}+J_{\tau^{\mathrm{b}}}^{-1}\right)^{-1}
$$

2) FIM of Location Parameters: To obtain the FIM of the location parameters (position and orientation), we need the EFIM of $\vartheta$ and $\tau$. Since the temporal and spatial parts in (17) are independent, the EFIM of DOD and DOA is obtained from (18) by Schur's complement

$$
\mathbf{J}_{\boldsymbol{\vartheta} \vartheta}^{\mathrm{e}, \mathrm{b}}=\mathbf{J}_{\boldsymbol{\vartheta} \vartheta}^{\mathrm{b}}-\frac{1}{J_{\beta_{\mathrm{R}} \beta_{\mathrm{R}}}^{\mathrm{b}}} \mathbf{J}_{\boldsymbol{\vartheta} \beta}^{\mathrm{b}}\left(\mathbf{J}_{\boldsymbol{\vartheta} \beta}^{\mathrm{b}}\right)^{\mathrm{T}} .
$$

Consequently, the EFIM of $\vartheta$ and $\tau$ is given by

$$
\mathbf{J}_{\boldsymbol{\vartheta} \tau}^{\mathrm{e}, \mathrm{b}}=\left[\begin{array}{cc}
\mathbf{J}_{\boldsymbol{\vartheta} \boldsymbol{\vartheta}}^{\mathrm{e}, \mathrm{b}} & \mathbf{0}_{4} \\
\mathbf{0}_{4}^{\mathrm{T}} & J_{\tau \tau}
\end{array}\right] \text {. }
$$

Applying a parameter transformation to (23), we obtain the EFIM of orientation and position

$$
\mathbf{J}_{\mathbf{o}, \mathbf{p}}^{\mathrm{e}, \mathrm{b}}=\boldsymbol{\Upsilon} \mathbf{J}_{\boldsymbol{\vartheta} \tau}^{\mathrm{e}, \mathrm{b}} \boldsymbol{\Upsilon}^{\mathrm{T}}
$$

where $\mathbf{\Upsilon} \triangleq\left[\begin{array}{l:l}\boldsymbol{\Upsilon}_{\mathrm{S}} & \boldsymbol{\Upsilon}_{\tau}\end{array}\right]$ is UL transformation matrix,

$$
\boldsymbol{\Upsilon} \triangleq\left[\begin{array}{llll:l}
\frac{\partial \theta_{\mathrm{B}}}{\partial \mathbf{o}} & \frac{\partial \phi_{\mathrm{B}}}{\partial \mathbf{o}} & \frac{\partial \theta_{\mathrm{U}}}{\partial \mathbf{o}} & \frac{\partial \phi_{\mathrm{U}}}{\partial \mathbf{o}} & \frac{\partial \tau}{\partial \mathbf{o}} \\
\frac{\partial \theta_{\mathrm{B}}}{\partial \mathbf{p}} & \frac{\partial \phi_{\mathrm{B}}}{\partial \mathbf{p}} & \frac{\partial \theta_{\mathrm{U}}}{\partial \mathbf{p}} & \frac{\partial \phi_{\mathrm{U}}}{\partial \mathbf{p}} & \frac{\partial \tau}{\partial \mathbf{p}}
\end{array}\right],
$$

such that [10]

$$
\begin{aligned}
\mathbf{\Upsilon}_{\mathrm{s}} & =\left[\begin{array}{cccc}
0 & 0 & -\frac{p_{y}^{\prime}}{a^{\prime}} & -\frac{p_{x}^{\prime} p_{z}^{\prime}}{a^{\prime 2}} \\
0 & 0 & \frac{p_{x}^{\prime} \sin \chi_{0}}{a^{\prime}} & \frac{-p_{x}^{\prime 2} \cos \chi_{0}+g p_{y}^{\prime}}{a^{\prime 2}} \\
\frac{\dot{\mathbf{p}}_{\theta}}{\|\mathbf{p}\| a} & \frac{\left[-p_{y}, p_{x}, 0\right]^{\mathrm{T}}}{a^{2}} & \frac{\mathbf{r}_{3}+\frac{p_{z}^{\prime}}{\|\mathbf{p}\| \mathbf{p}}}{a^{\prime}} & \frac{\left(\mathbf{r}_{\mathrm{U}} \mathbf{r}_{\mathrm{B}}^{\mathrm{T}}-\mathbf{r}_{\mathrm{B}} \mathbf{r}_{\mathrm{U}}^{\mathrm{T}}\right) \mathbf{p}}{a^{\prime 2}}
\end{array}\right] \\
\mathbf{\Upsilon}_{\tau} & =\left[\begin{array}{ll}
\mathbf{0}_{\mathrm{U}}^{\mathrm{T}} & \frac{\mathbf{p}^{\mathrm{T}}}{c\|\mathbf{p}\|}
\end{array}\right]^{\mathrm{T}},
\end{aligned}
$$


where $g \triangleq p_{y} \cos \zeta_{0}-p_{x} \sin \zeta_{0}, \quad \dot{\mathbf{p}}_{\theta} \triangleq$ $\left[p_{x} p_{z}, p_{y} p_{z},-a^{2}\right]^{\mathrm{T}}, a \triangleq \sqrt{p_{x}^{2}+p_{y}^{2}}, a^{\prime} \triangleq \sqrt{p_{x}^{\prime 2}+p_{y}^{\prime 2}}$, $\left[p_{x}^{\prime}, p_{y}^{\prime}, p_{z}^{\prime}\right]^{\mathrm{T}} \triangleq \mathbf{R} \mathbf{p}$, and $\mathbf{R} \triangleq\left[\mathbf{r}_{1}, \mathbf{r}_{2}, \mathbf{r}_{3}\right]=$

$$
\left[\begin{array}{ccc}
\cos \zeta_{0} & -\sin \zeta_{0} \cos \chi_{0} & -\sin \zeta_{0} \sin \chi_{0} \\
\sin \zeta_{0} & \cos \zeta_{0} \cos \chi_{0} & \cos \zeta_{0} \sin \chi_{0} \\
0 & -\sin \chi_{0} & \cos \chi_{0}
\end{array}\right] .
$$

Subsequently, for DLP, we can isolate the spatial and temporal parts and write,

$$
\mathbf{J}_{\mathbf{o}, \mathbf{p}}^{\mathrm{e}, \mathrm{b}}=\underbrace{\mathbf{\Upsilon}_{\mathrm{s}} \mathbf{J}_{\vartheta \vartheta}^{\mathrm{e}, \mathrm{b}} \mathbf{\Upsilon}_{\mathrm{s}}^{\mathrm{T}}}_{\text {Spatial Part }}+\underbrace{J_{\tau \tau} \mathbf{\Upsilon}_{\tau} \mathbf{\Upsilon}_{\tau}^{\mathrm{T}}}_{\text {Temporal Part }} .
$$

3) Performance Comparison of DLP with OWL: To compare DLP with OWL, recall that for OWL, $\mathbf{J}_{\mathbf{o}, \mathbf{p}}^{\mathrm{e}, \mathrm{b}}$ has the same expression as (27), but with $J_{\tau \tau}=J_{\tau^{\mathrm{b}}}$. Therefore, in comparison with (21), DLP outperforms OWL when

$$
J_{\tau^{\mathrm{b}}}<4\left(J_{\tau^{\mathrm{f}}}^{-1}+J_{\tau^{\mathrm{b}}}^{-1}\right)^{-1}=J_{\tau^{\mathrm{b}}} \frac{4 J_{\tau^{\mathrm{f}}}}{J_{\tau^{\mathrm{f}}}+J_{\tau^{\mathrm{b}}}},
$$

which leads to $J_{\tau^{\mathrm{f}}}>\frac{1}{3} J_{\tau^{\mathrm{b}}}$.

This means that, when the bandwidth is equal in both directions, the forward link should have at least one third the SNR of the backward link for DLP to outperform OWL. From Appendix A in [13], it can be seen that this mainly depends on the trasnmit and receive beamformin.

\section{Simulation Results and Discussion}

In this section, we numerically investigate and compare the DLP and OWL. Since DLP involves forward and backward transmission, we select equal number of antennas at the BS and the UE to make a fair comparison. Towards that, we consider a BS and a UE both with $12 \times 12$ uniform rectangular antenna array communicating via a LOS. Moreover, we assume that the BS array is located in the $x z$-plane centered about the 3D origin, thus has orientation of $\left[0^{\circ}, 0^{\circ}\right]^{\mathrm{T}}$. The UE is located in a diamond shape area defined by $(0,0,-10),(25 \sqrt{3}, 25,-10),(0,50,-10)$, and $(-25 \sqrt{3}, 25,-10)$. That is, the BS height is 10 meters. We focus on two cases of orientation: $\mathbf{o}=\left[0^{\circ}, 0^{\circ}\right]^{\mathrm{T}}$ and $\mathbf{o}=\left[15^{\circ}, 15^{\circ}\right]^{\mathrm{T}}$. Finally, at a distance $d_{0}$, the channel gain is modeled as

$$
\beta=\frac{\lambda}{4 \pi d_{0}} \mathrm{e}^{j \frac{2 \pi d_{0}}{\lambda}}
$$

We select $f=38 \mathrm{GHz}$, and $W=125 \mathrm{MHz}$, transmitted power $E_{\mathrm{t}} / T_{\mathrm{s}}=0 \mathrm{dBm}, N_{0}=-170 \mathrm{dBm} / \mathrm{Hz}$, and $N_{\mathrm{s}}=64$ pilot symbols. We further assume an ideal sinc pulse-shaping filter with $W_{\text {eff }}^{2}=W^{2} / 3$. Similar to [10], we adopt fixed directional beamforming with $N_{\mathrm{b}_{\mathrm{B}}}=N_{\mathrm{bU}}=25$ such that

$$
\begin{aligned}
\mathbf{f}_{\mathrm{B}, b} & =\frac{1}{\sqrt{N_{\mathrm{b}_{\mathrm{B}}}}} \mathbf{a}_{\mathrm{B}}\left(\theta_{\mathrm{B}, b}^{\mathrm{f}}, \phi_{\mathrm{B}, b}^{\mathrm{f}}\right), \\
\mathbf{w}_{\mathrm{B}, b} & =\frac{1}{\sqrt{N_{\mathrm{b}_{\mathrm{B}}}}} \mathbf{a}_{\mathrm{B}}\left(\theta_{\mathrm{B}, b}^{\mathrm{w}}, \phi_{\mathrm{B}, b}^{\mathrm{w}}\right), \quad 1 \leq b \leq N_{\mathrm{b}_{\mathrm{B}}},
\end{aligned}
$$

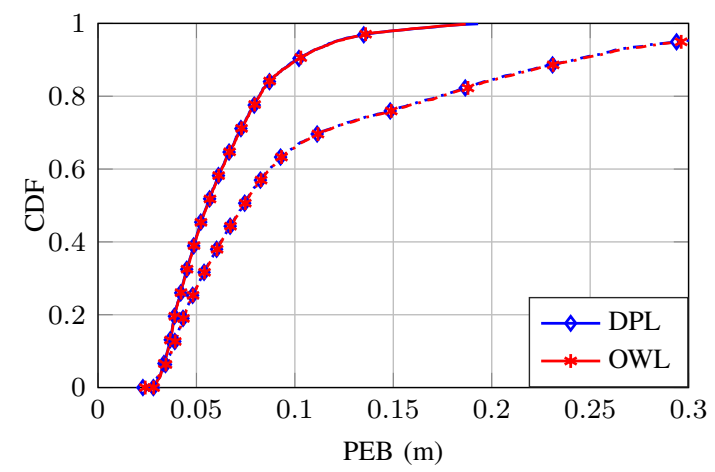

Fig. 3. CDF of PEB with UE orientation angles of $0^{\circ}$ (solid) and $15^{\circ}$ (dash-dot). $N_{\mathrm{U}}=N_{\mathrm{B}}=144, N_{\mathrm{B}}=25$.

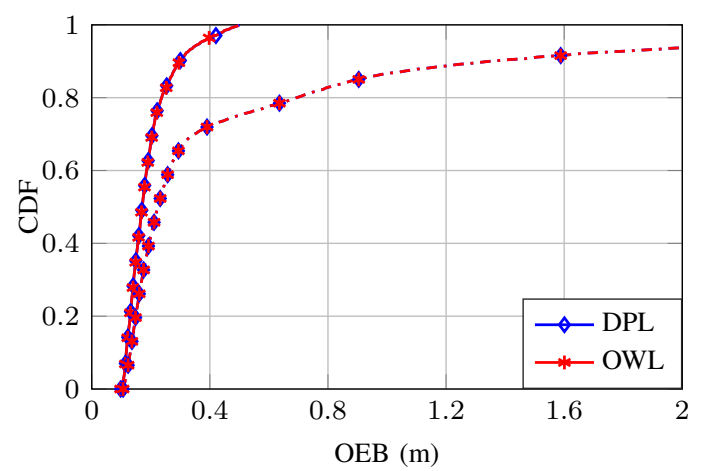

Fig. 4. CDF of OEB with UE orientation angles of $0^{\circ}$ (solid) and $15^{\circ}$ (dash-dot). $N_{\mathrm{U}}=N_{\mathrm{B}}=144, N_{\mathrm{B}}=25$.

are BS transmit and receive beams pointing towards $\left(\theta_{\mathrm{B}, b}^{\mathrm{f}}, \phi_{\mathrm{B}, b}^{\mathrm{f}}\right)$ and $\left(\theta_{\mathrm{B}, b}^{\mathrm{w}}, \phi_{\mathrm{B}, b}^{\mathrm{w}}\right)$, respectively. The transmit and receive beamforming at UE can be similarly defined. The beam directions at the BS are chosen to be equispaced on the sector, while at the UE, they are reversed to point upwards, rotated with respect to the UE frame of reference by the same orientation angles specified in the studied experiment. Consequently, for $i, j \in\{\mathrm{B}, \mathrm{U}\}, i \neq j$, the resulting SNR is

$$
\mathrm{SNR}[\mathrm{dB}]=150.26+20 \log _{\mathrm{B} 0}\left(|\beta|\left\|\mathbf{a}_{i} \mathbf{F}_{i}\right\|\left\|\mathbf{a}_{j} \mathbf{W}_{j}\right\|\right) \text {. }
$$

\section{A. PEB with $0^{\circ}$ and $15^{\circ}$ UE Orientation}

The PEB with zero orientation angles is provided in Fig. 3 (solid lines). It can be seen that DLP provides no improvement over OWL in the considered setup. Despite that, DLP is still a better approach since it alleviates the need of tight synchronization, with the added cost of UE-BS coordination. As discussed in Section IV-3, DLP and OWL have the same spatial component, but DLP has higher temporal information content. However, Fig. 3 shows almost identical results for both protocols, which means that the additional temporal information in DLP is of little importance, and hence the localization performance is limited by the angles estimation rather than the estimation of the time delay. More on this issue will be discussed in Section V-C.

The PEB with $\mathbf{o}=\left[15^{\circ}, 15^{\circ}\right]^{\mathrm{T}}$ is denoted by the dash-dot lines in Fig. 3. The overall observation from this figure is that the performance worsens as beams steer away, leading to UE mis-orientation with respect 


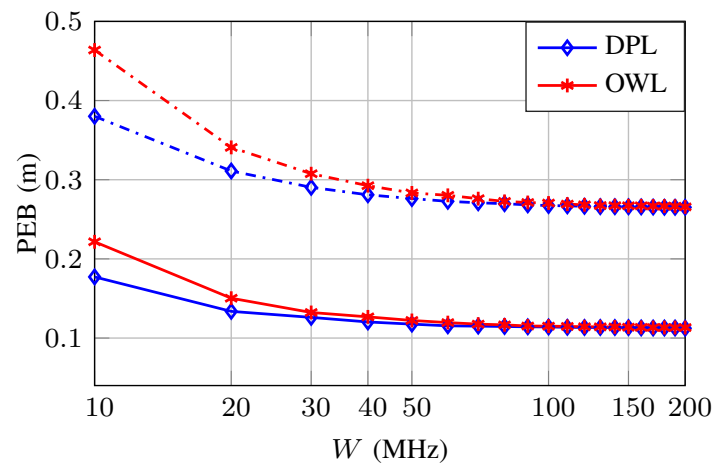

Fig. 5. PEB at $0.9 \mathrm{CDF}$ with respect to the bandwidth $W$, with UE orientation angles of $0^{\circ}$ (solid) and $15^{\circ}$ (dash-dot).

to BS. This can result in a loss of beamforming gain that depends non-linearly on the UE location.

\section{B. OEB with $0^{\circ}$ and $15^{\circ}$ UE Orientation}

Considering $\mathrm{OEB}$ with $0^{\circ}$ and $15^{\circ} \mathrm{UE}$ orientation in Fig. 4, it can be seen that DLP and OWL exhibit identical performance. Note that OEB depends on DOA and DOD, while the enhancement of DLP over OWL is in the temporal domain. Moreover, in comparison with the case of matched orientation, the system can still provide submeter PEB under $15^{\circ}$ mis-orientation, while providing significantly higher OEB. This means that orientation estimation is more challenging than position estimation.

\section{Impact of the System Bandwidth on PEB}

In Section V-A, we concluded that the system is limited by the estimation of the angles rather than the time delay. To investigate this further, we now look closer into the impact of the bandwidth. The results shown in Fig. 5 indicate that as the bandwidth increases, the PEB decreases before reaching a floor at around 100 $\mathrm{MHz}$, which leads to the following observations.

Firstly, at the high bandwidths relevant in mmWave, the temporal information is very high compared to the spatial information, and the performance becomes fixed with $W$, leading the systems to be spatially-limited. Moreover, under mis-orientation, the accuracy of spatial information degrades, and the improved temporal information does not provide any benefit to the performance achieved at lower bandwidths. Furthermore, at lower bandwidths, the amount of temporal information decreases and becomes comparable to the spatial information. Therefore, the weight of the temporal information in the forward transmission becomes more significant, and the OWL and DLP diverge.

\section{Impact of $N_{\mathrm{B}}$ and $N_{\mathrm{U}}$ on $P E B$}

We now study the effect of the number of antennas at BS and UE on the PEB under DLP. Fig. 6 illustrates the effect of $N_{\mathrm{U}}$ and $N_{\mathrm{B}}$ on PEB. Note that the PEB curves with respect to $N_{\mathrm{U}}$ are obtained with a fixed $N_{\mathrm{B}}=144$, and vice versa. It can be seen from Fig. 6 that a higher $N_{\mathrm{U}}$ generally results in a worse performance. This is because with higher $N_{\mathrm{U}}$, the UE beams become narrower, which requires more beams to cover the area.

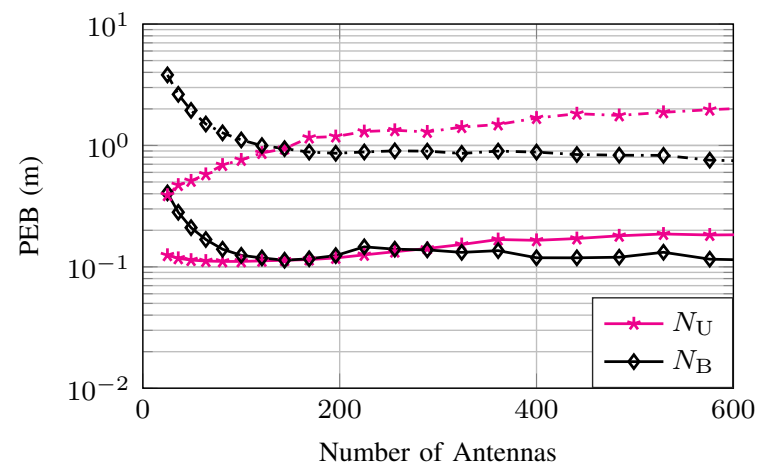

Fig. 6. PEB at $0.9 \mathrm{CDF}$ as a function of $N_{\mathrm{U}}$ and $N_{\mathrm{B}}$ for $N_{\mathrm{b}_{\mathrm{B}}}=25$, with UE orientation angles of $0^{\circ}$ (solid) and $15^{\circ}$ (dash-dot). PEB curves w.r.t $N_{\mathrm{U}}$ are obtained with $N_{\mathrm{B}}=144$, while those w.r.t $N_{\mathrm{B}}$ are obtained with $N_{\mathrm{U}}=144$

Moreover, it can be deduced that a higher $N_{\mathrm{B}}$ will slightly improve the PEB in general. Similar to the case in of $N_{\mathrm{U}}$, it is understood that the PEB will generally increase when $N_{\mathrm{B}}$ increases, albeit, at $N_{\mathrm{B}}$ values well beyond those displayed in Fig. 6, and with a lesser magnitude than higher $N_{\mathrm{U}}$. Therefore, adding more antennas at the BS will not reduce the localization performance, as the UE antennas potentially would, at least within the studied range of array size.

Finally, notice that Fig. 6 exhibits some nonmonotonic trend. This is due to the nature of directional beamforming, whereby the beamforming gain depends on the user location, number of antennas, and beams directions as detailed in [14].

\section{CONCLUSIONS}

Many papers on localization assume that the BS and UE are tightly synchronized. However, usually communication is not synchronized to a high-level useful for localization. Focusing on this issue, in this paper, we considered distributed localization protocol (DLP) and investigated the corresponding PEB and OEB. From the results of the numerical simulation, there was no enhancement observed for DLP over the traditional oneway localization in terms of PEB and OEB. That is, the localization was angle-limited rather than delaylimited. For future work, multipath propagation would be a relevant extension, since scatterers may differ in the uplink and downlink, depending on the beam directions.

\section{ACKNOWLEDGMENT}

The authors wish to thank Dr. Xiagyun Zhou of the Australian National University for his valuable feedback.

This work is supported in part by the Australian Government's Research Training Program (RTP), the Horizon2020 projects HIGHTS (High precision positioning for cooperative ITS applications) MG-3.5a-2014636537 and 5GCAR (Fifth Generation Communication Automotive Research and innovation), the VINNOVA COPPLAR project, funded under Strategic Vehicle Research and Innovation Grant No. 2015-04849, and the Spanish Ministry of Economy, Industry and Competitiveness under Grant TEC2017-89925-R. 


\section{APPENDIX \\ Derivation of THE Elements OF $\mathbf{J}_{\varphi_{\mathrm{D}}}$}

From (18), we define

$$
\mathbf{J}_{\vartheta \vartheta}^{\mathrm{b}} \triangleq\left[\begin{array}{cccc}
J_{\theta_{\mathrm{B}} \theta_{\mathrm{B}}}^{\mathrm{b}} & J_{\theta_{\mathrm{B}} \phi_{\mathrm{B}}}^{\mathrm{b}} & J_{\theta_{\mathrm{B}} \theta_{\mathrm{U}}}^{\mathrm{b}} & J_{\theta_{\mathrm{B}} \phi_{\mathrm{U}}}^{\mathrm{b}} \\
J_{\theta_{\mathrm{B}} \phi_{\mathrm{B}}}^{\mathrm{b}} & J_{\phi_{\mathrm{B}} \phi_{\mathrm{B}}}^{\mathrm{b}} & J_{\phi_{\mathrm{B}} \theta_{\mathrm{U}}}^{\mathrm{b}} & J_{\phi_{\mathrm{B}} \phi_{\mathrm{U}}}^{\mathrm{b}} \\
J_{\theta_{\mathrm{B}} \theta_{\mathrm{U}}}^{\mathrm{b}} & J_{\phi_{\mathrm{B}} \theta_{\mathrm{U}}}^{\mathrm{b}} & J_{\theta_{\mathrm{U}} \theta_{\mathrm{U}}}^{\mathrm{b}} & J_{\theta_{\mathrm{U}} \phi_{\mathrm{U}}}^{\mathrm{b}} \\
J_{\theta_{\mathrm{B}} \phi_{\mathrm{U}}}^{\mathrm{b}} & J_{\phi_{\mathrm{B}} \phi_{\mathrm{U}}}^{\mathrm{b}} & J_{\theta_{\mathrm{U}} \phi_{\mathrm{U}}}^{\mathrm{b}} & J_{\phi_{\mathrm{U}} \phi_{\mathrm{U}}}^{\mathrm{b}}
\end{array}\right]
$$

and

$$
\left(\mathbf{J}_{\boldsymbol{\vartheta} \beta}^{\mathrm{b}}\right)^{\mathrm{T}} \triangleq\left[\begin{array}{cccc}
J_{\theta_{\mathrm{B}} \beta_{\mathrm{R}}}^{\mathrm{b}} & J_{\phi_{\mathrm{B}} \beta_{\mathrm{R}}}^{\mathrm{b}} & J_{\theta_{\mathrm{U}} \beta_{\mathrm{R}}}^{\mathrm{b}} & J_{\phi_{\mathrm{U}} \beta_{\mathrm{R}}}^{\mathrm{b}} \\
J_{\theta_{\mathrm{B}} \beta_{\mathrm{I}}}^{\mathrm{b}} & J_{\phi_{\mathrm{B}} \beta_{\mathrm{I}}}^{\mathrm{b}} & J_{\theta_{\mathrm{U}} \beta_{\mathrm{I}}}^{\mathrm{b}} & J_{\phi_{\mathrm{U}} \beta_{\mathrm{I}}}^{\mathrm{b}}
\end{array}\right]
$$

For the case of zero-mean additive correlated Gaussian noise, the FIM of $\varphi_{\mathrm{D}}$ defined in (16), is given by [15]

$$
J_{x y}^{\mathrm{b}} \triangleq \frac{1}{N_{0}} \int_{0}^{T_{\mathrm{o}}} \Re\left\{\frac{\partial \boldsymbol{\mu}^{\mathrm{H}}(t)}{\partial x}\left(\mathbf{W}_{\mathrm{B}}^{\mathrm{H}} \mathbf{W}_{\mathrm{B}}\right)^{-1} \frac{\partial \boldsymbol{\mu}(t)}{\partial y}\right\} \mathrm{d} t
$$

where $x, y \in\left\{\theta_{\mathrm{B}}, \phi_{\mathrm{B}}, \theta_{\mathrm{U}}, \phi_{\mathrm{U}}, \beta_{\mathrm{R}}, \beta_{\mathrm{I}}, \tau\right\}$, and $\boldsymbol{\mu}(t)$ is the mean of the observation vector in (11), and $T_{\mathrm{O}}$ is assumed to be long enough to receive the entire pilot signal. That is,

$$
\boldsymbol{\mu}(t)=\sqrt{N_{\mathrm{B}} N_{\mathrm{U}} E_{\mathrm{t}}} \beta \mathbf{W}_{\mathrm{B}}^{\mathrm{H}} \mathbf{a}_{\mathrm{B}} \mathbf{a}_{\mathrm{U}}^{\mathrm{H}} \mathbf{F}_{\mathrm{U}} \mathbf{s}_{\mathrm{U}}\left(t-\tau^{\mathrm{b}}\right) .
$$

Differentiating $\boldsymbol{\mu}(t)$ w.r.t channel parameters and substituting in (31), while defining $\mathbf{P}_{\mathbf{A}} \triangleq \mathbf{A}\left(\mathbf{A}^{\mathrm{H}} \mathbf{A}\right)^{-1} \mathbf{A}^{\mathrm{H}}$, and $\gamma \triangleq N_{\mathrm{B}} N_{\mathrm{U}} N_{\mathrm{s}} E_{\mathrm{t}} / N_{0}$, it can be shown that

$$
\begin{aligned}
& J_{\theta_{\mathrm{B}} \theta_{\mathrm{B}}}^{\mathrm{b}}=\gamma|\beta|^{2}\left(\mathbf{a}_{\mathrm{U}}^{\mathrm{H}} \mathbf{F}_{\mathrm{U}} \mathbf{F}_{\mathrm{U}}^{\mathrm{H}} \mathbf{a}_{\mathrm{U}}\right)\left(\mathbf{k}_{\mathrm{B}}^{\mathrm{H}} \mathbf{P}_{\mathbf{W}_{\mathrm{B}}} \mathbf{k}_{\mathrm{B}}\right) \\
& J_{\phi_{\mathrm{B}} \phi_{\mathrm{B}}}^{\mathrm{b}}=\gamma|\beta|^{2}\left(\mathbf{a}_{\mathrm{U}}^{\mathrm{H}} \mathbf{F}_{\mathrm{U}} \mathbf{F}_{\mathrm{U}}^{\mathrm{H}} \mathbf{a}_{\mathrm{U}}\right)\left(\mathbf{p}_{\mathrm{B}}^{\mathrm{H}} \mathbf{P}_{\mathbf{W}_{\mathrm{B}}} \mathbf{p}_{\mathrm{B}}\right) \\
& J_{\theta_{\mathrm{U}} \theta_{\mathrm{U}}}^{\mathrm{b}}=\gamma|\beta|^{2}\left(\mathbf{k}_{\mathrm{U}}^{\mathrm{H}} \mathbf{F}_{\mathrm{U}} \mathbf{F}_{\mathrm{U}}^{\mathrm{H}} \mathbf{k}_{\mathrm{U}}\right)\left(\mathbf{a}_{\mathrm{B}}^{\mathrm{H}} \mathbf{P}_{\mathbf{w}_{\mathrm{B}}} \mathbf{a}_{\mathrm{B}}\right) \\
& J_{\phi_{\mathrm{U}} \phi_{\mathrm{U}}}^{\mathrm{b}}=\gamma|\beta|^{2}\left(\mathbf{p}_{\mathrm{U}}^{\mathrm{H}} \mathbf{F}_{\mathrm{U}} \mathbf{F}_{\mathrm{U}}^{\mathrm{H}} \mathbf{p}_{\mathrm{U}}\right)\left(\mathbf{a}_{\mathrm{B}}^{\mathrm{H}} \mathbf{P}_{\mathbf{W}_{\mathrm{B}}} \mathbf{a}_{\mathrm{B}}\right) \\
& J_{\beta_{\mathrm{R}} \beta_{\mathrm{R}}}^{\mathrm{b}}=J_{\beta_{\mathrm{I}} \beta_{\mathrm{I}}}^{\mathrm{b}} \text {, } \\
& =\gamma|\beta|^{2}\left(\mathbf{a}_{\mathrm{U}}^{\mathrm{H}} \mathbf{F}_{\mathrm{U}} \mathbf{F}_{\mathrm{U}}^{\mathrm{H}} \mathbf{a}_{\mathrm{R} 2}\right)\left(\mathbf{a}_{\mathrm{B}}^{\mathrm{H}} \mathbf{P}_{\mathbf{w}_{\mathrm{B}}} \mathbf{a}_{\mathrm{B}}\right), \\
& J_{\theta_{\mathrm{B}} \phi_{\mathrm{B}}}^{\mathrm{b}}=\gamma|\beta|^{2}\left(\mathbf{a}_{\mathrm{U}}^{\mathrm{H}} \mathbf{F}_{\mathrm{U}} \mathbf{F}_{\mathrm{U}}^{\mathrm{H}} \mathbf{a}_{\mathrm{U}}\right)\left(\mathbf{p}_{\mathrm{B}}^{\mathrm{H}} \mathbf{P}_{\mathbf{W}_{\mathrm{B}}} \mathbf{k}_{\mathrm{B}}\right) \text {, } \\
& J_{\theta_{\mathrm{B}} \theta_{\mathrm{U}}}^{\mathrm{b}}=\gamma|\beta|^{2}\left(\mathbf{k}_{\mathrm{U}}^{\mathrm{H}} \mathbf{F}_{\mathrm{U}} \mathbf{F}_{\mathrm{U}}^{\mathrm{H}} \mathbf{a}_{\mathrm{U}}\right)\left(\mathbf{k}_{\mathrm{B}}^{\mathrm{H}} \mathbf{P}_{\mathbf{w}_{\mathrm{B}}} \mathbf{a}_{\mathrm{B}}\right), \\
& J_{\theta_{\mathrm{B}} \phi_{\mathrm{U}}}^{\mathrm{b}}=\gamma|\beta|^{2}\left(\mathbf{p}_{\mathrm{U}}^{\mathrm{H}} \mathbf{F}_{\mathrm{U}} \mathbf{F}_{\mathrm{U}}^{\mathrm{H}} \mathbf{a}_{\mathrm{U}}\right)\left(\mathbf{k}_{\mathrm{B}}^{\mathrm{H}} \mathbf{P}_{\mathbf{W}_{\mathrm{B}}} \mathbf{a}_{\mathrm{B}}\right), \\
& J_{\theta_{\mathrm{B}} \beta_{\mathrm{R}}}^{\mathrm{b}}=\gamma \Re\left[\beta^{*}\left(\mathbf{a}_{\mathrm{U}}^{\mathrm{H}} \mathbf{F}_{\mathrm{U}} \mathbf{F}_{\mathrm{U}}^{\mathrm{H}} \mathbf{a}_{\mathrm{U}}\right)\left(\mathbf{k}_{\mathrm{B}}^{\mathrm{H}} \mathbf{P}_{\mathbf{W}_{\mathrm{B}}} \mathbf{a}_{\mathrm{B}}\right)\right], \\
& J_{\theta_{\mathrm{B}} \beta_{\mathrm{I}}}^{\mathrm{b}}=-\gamma \Im\left[\beta^{*}\left(\mathbf{a}_{\mathrm{U}}^{\mathrm{H}} \mathbf{F}_{\mathrm{U}} \mathbf{F}_{\mathrm{U}}^{\mathrm{H}} \mathbf{a}_{\mathrm{U}}\right)\left(\mathbf{k}_{\mathrm{B}}^{\mathrm{H}} \mathbf{P}_{\mathbf{w}_{\mathrm{B}}} \mathbf{a}_{\mathrm{B}}\right)\right], \\
& J_{\phi_{\mathrm{B}} \theta_{\mathrm{U}}}^{\mathrm{b}}=\gamma|\beta|^{2}\left(\mathbf{k}_{\mathrm{U}}^{\mathrm{H}} \mathbf{F}_{\mathrm{U}} \mathbf{F}_{\mathrm{U}}^{\mathrm{H}} \mathbf{a}_{\mathrm{U}}\right)\left(\mathbf{p}_{\mathrm{B}}^{\mathrm{H}} \mathbf{P}_{\mathbf{W}_{\mathrm{B}}} \mathbf{a}_{\mathrm{B}}\right) \text {, } \\
& J_{\phi_{\mathrm{B}} \phi_{\mathrm{U}}}^{\mathrm{b}}=\gamma|\beta|^{2}\left(\mathbf{p}_{\mathrm{U}}^{\mathrm{H}} \mathbf{F}_{\mathrm{U}} \mathbf{F}_{\mathrm{U}}^{\mathrm{H}} \mathbf{a}_{\mathrm{U}}\right)\left(\mathbf{p}_{\mathrm{B}}^{\mathrm{H}} \mathbf{P}_{\mathbf{W}_{\mathrm{B}}} \mathbf{a}_{\mathrm{B}}\right), \\
& J_{\phi_{\mathrm{B}} \beta_{\mathrm{R}}}^{\mathrm{b}}=\gamma \Re\left[\beta^{*}\left(\mathbf{a}_{\mathrm{U}}^{\mathrm{H}} \mathbf{F}_{\mathrm{U}} \mathbf{F}_{\mathrm{U}}^{\mathrm{H}} \mathbf{a}_{\mathrm{U}}\right)\left(\mathbf{p}_{\mathrm{B}}^{\mathrm{H}} \mathbf{P}_{\mathbf{W}_{\mathrm{B}}} \mathbf{a}_{\mathrm{B}}\right)\right], \\
& J_{\phi_{\mathrm{B}} \beta_{\mathrm{I}}}^{\mathrm{b}}=-\gamma \Im\left[\beta^{*}\left(\mathbf{a}_{\mathrm{U}}^{\mathrm{H}} \mathbf{F}_{\mathrm{U}} \mathbf{F}_{\mathrm{U}}^{\mathrm{H}} \mathbf{a}_{\mathrm{U}}\right)\left(\mathbf{p}_{\mathrm{B}}^{\mathrm{H}} \mathbf{P}_{\mathbf{W}_{\mathrm{B}}} \mathbf{a}_{\mathrm{B}}\right)\right], \\
& J_{\theta_{\mathrm{U}} \phi_{\mathrm{U}}}^{\mathrm{b}}=\gamma|\beta|^{2}\left(\mathbf{p}_{\mathrm{U}}^{\mathrm{H}} \mathbf{F}_{\mathrm{U}} \mathbf{F}_{\mathrm{U}}^{\mathrm{H}} \mathbf{k}_{\mathrm{U}}\right)\left(\mathbf{a}_{\mathrm{B}}^{\mathrm{H}} \mathbf{P}_{\mathbf{W}_{\mathrm{B}}} \mathbf{a}_{\mathrm{B}}\right) \text {, } \\
& J_{\theta_{\mathrm{U}} \beta_{\mathrm{R}}}^{\mathrm{b}}=\gamma \Re\left[\beta^{*}\left(\mathbf{a}_{\mathrm{U}}^{\mathrm{H}} \mathbf{F}_{\mathrm{U}} \mathbf{F}_{\mathrm{U}}^{\mathrm{H}} \mathbf{k}_{\mathrm{U}}\right)\left(\mathbf{a}_{\mathrm{B}}^{\mathrm{H}} \mathbf{P}_{\mathbf{w}_{\mathrm{B}}} \mathbf{a}_{\mathrm{B}}\right)\right], \\
& J_{\theta_{\mathrm{U}} \beta_{\mathrm{I}}}^{\mathrm{b}}=-\gamma \Im\left[\beta^{*}\left(\mathbf{a}_{\mathrm{U}}^{\mathrm{H}} \mathbf{F}_{\mathrm{U}} \mathbf{F}_{\mathrm{U}}^{\mathrm{H}} \mathbf{k}_{\mathrm{U}}\right)\left(\mathbf{a}_{\mathrm{B}}^{\mathrm{H}} \mathbf{P}_{\mathbf{W}_{\mathrm{B}}} \mathbf{a}_{\mathrm{B}}\right)\right], \\
& J_{\phi_{\mathrm{U}} \beta_{\mathrm{R}}}^{\mathrm{b}}=\gamma \Re\left[\beta^{*}\left(\mathbf{a}_{\mathrm{U}}^{\mathrm{H}} \mathbf{F}_{\mathrm{U}} \mathbf{F}_{\mathrm{U}}^{\mathrm{H}} \mathbf{p}_{\mathrm{U}}\right)\left(\mathbf{a}_{\mathrm{B}}^{\mathrm{H}} \mathbf{P}_{\mathbf{W}_{\mathrm{B}}} \mathbf{a}_{\mathrm{B}}\right)\right],
\end{aligned}
$$

$$
J_{\phi_{\mathrm{U}} \beta_{\mathrm{I}}}^{\mathrm{b}}=-\gamma \Im\left[\beta^{*}\left(\mathbf{a}_{\mathrm{U}}^{\mathrm{H}} \mathbf{F}_{\mathrm{U}} \mathbf{F}_{\mathrm{U}}^{\mathrm{H}} \mathbf{p}_{\mathrm{U}}\right)\left(\mathbf{a}_{\mathrm{B}}^{\mathrm{H}} \mathbf{P}_{\mathbf{w}_{\mathrm{B}}} \mathbf{a}_{\mathrm{B}}\right)\right] .
$$

where $\mathbf{k}_{\mathrm{i}}=\frac{\partial}{\partial \theta_{i}} \mathbf{a}_{\mathbf{i}}, \mathbf{p}_{\mathrm{i}}=\frac{\partial}{\partial \phi_{i}} \mathbf{a}_{\mathrm{i}}$, such that $i \in\{\mathrm{B}, \mathrm{U}\}$. To compute $J_{\tau \tau}$, in (21), we extend the results in [10] to

$$
\begin{aligned}
J_{\tau^{\mathrm{b}}} & =\frac{1}{4 \gamma|\beta|^{2} \pi^{2} W_{\mathrm{eff}}^{2}\left\|\mathbf{a}_{\mathrm{U}}^{\mathrm{H}} \mathbf{F}_{\mathrm{U}}\right\|^{2}\left(\mathbf{a}_{\mathrm{B}}^{\mathrm{H}} \mathbf{P}_{\mathbf{W}_{\mathrm{B}}} \mathbf{a}_{\mathrm{B}}\right)}, \\
J_{\tau^{\mathrm{f}}} & =\frac{1}{4 \gamma|\beta|^{2} \pi^{2} W_{\mathrm{eff}}^{2}\left\|\mathbf{a}_{\mathrm{B}}^{\mathrm{H}} \mathbf{F}_{\mathrm{B}}\right\|^{2}\left(\mathbf{a}_{\mathrm{U}}^{\mathrm{H}} \mathbf{P}_{\mathbf{W}_{\mathrm{U}}} \mathbf{a}_{\mathrm{U}}\right)},
\end{aligned}
$$

where,

$$
W_{\mathrm{eff}}^{2}=\int_{-W / 2}^{W / 2} f^{2}|P(f)|^{2} \mathrm{~d} f
$$

\section{REFERENCES}

[1] N. Garcia, H. Wymeersch, E. G. Ström, and D. Slock, "Locationaided mm-wave channel estimation for vehicular communication," in IEEE 17th Int. Workshop on Signal Process. Advances in Wireless Commun., July 2016, pp. 1-5.

[2] K. Witrisal, P. Meissner, E. Leitinger, Y. Shen, C. Gustafson, F. Tufvesson, K. Haneda, D. Dardari, A. F. Molisch, A. Conti, and M. Z. Win, "High-accuracy localization for assisted living: 5G systems will turn multipath channels from foe to friend," IEEE Signal Process. Mag, vol. 33, no. 2, pp. 59-70, March 2016.

[3] J. C. Aviles and A. Kouki, "Position-aided mm-wave beam training under NLOS conditions," IEEE Access, vol. 4, pp. 87038714, 2016.

[4] N. Akbar, S. Yan, N. Yang, and J. Yuan, "Mitigating pilot contamination through location-aware pilot assignment in massive MIMO networks," in 2016 IEEE Globecom Workshops (GC Wkshps), Dec 2016, pp. 1-6.

[5] L. S. Muppirisetty, T. Svensson, and H. Wymeersch, "Spatial wireless channel prediction under location uncertainty," IEEE Trans. on Wireless Commun., vol. 15, no. 2, pp. 1031-1044, Feb 2016.

[6] M. D. Larsen, A. L. Swindlehurst, and T. Svantesson, "Performance bounds for MIMO-OFDM channel estimation," IEEE Trans. Signal Process., vol. 57, no. 5, pp. 1901-1916, May 2009.

[7] Y. Shen, H. Wymeersch, and M. Z. Win, "Fundamental limits of wideband localization - part II: Cooperative networks," IEEE Trans. on Info Theory, vol. 56, no. 10, pp. 4981-5000, Oct 2010.

[8] A. Shahmansoori, G. E. Garcia, G. Destino, G. Seco-Granados, and $\mathrm{H}$. Wymeersch, "Position and orientation estimation through millimeter-wave mimo in 5G systems," IEEE Transactions on Wireless Communications, vol. 17, no. 3, pp. 1822-1835, March 2018.

[9] A. Guerra, F. Guidi, and D. Dardari, "Single anchor localization and orientation performance limits using massive arrays: MIMO vs. beamforming," 2017. [Online]. Available: https://arxiv.org/abs/1702.01670

[10] Z. Abu-Shaban, X. Zhou, T. Abhayapala, G. Seco-Granados, and H. Wymeersch, "Error bounds for uplink and downlink 3D localization in 5G mmwave systems," Accepted at IEEE Transactions on Wireless Communications, April 2018.

[11] Z. Sahinoglu, S. Gezici, and I. Guvenc, Ultra-wideband positioning systems : theoretical limits, ranging algorithms, and protocols. Cambridge University Press, 2008.

[12] N. A. H. Duisterwinkel, Erik H. A.and Puts and H. J. Wörtche, "Asymmetric multi-way ranging for resource-limited nodes," in Ad Hoc Networks, Y. Zhou and T. Kunz, Eds. Springer International Publishing, 2017, pp. 50-63.

[13] Z. Abu-Shaban, H. Wymeersch, T. Abhayapala, and G. SecoGranados, "Single-anchor two-way localization bounds for $5 \mathrm{G}$ mmwave systems: Two protocols," 2018. [Online]. Available: https://arxiv.org/abs/1805.02319

[14] Z. Abu-Shaban, H. Wymeersch, X. Zhou, G. Seco-Granados, and T. Abhayapala, "Random-phase beamforming for initial access in millimeter-wave cellular networks," in 2016 IEEE GLOBECOM Conf., Dec 2016, pp. 1-6.

[15] S. M. Kay, Fundamentals of Statistical Signal Processing: Estimation Theory. NJ, USA: Prentice-Hall, Inc., 1993. 